\title{
Basic Problems in Multi-View Modeling ${ }^{\star}$
}

\author{
Jan Reineke $^{1}$ and Stavros Tripakis ${ }^{2}$ \\ 1 Saarland University \\ ${ }^{2}$ UC Berkeley and Aalto University
}

\begin{abstract}
Modeling all aspects of a complex system within a single model is a difficult, if not impossible, task. Multi-view modeling is a methodology where different aspects of the system are captured by different models, or views. A key question then is consistency: if different views of a system have some degree of overlap, how can we guarantee that they are consistent, i.e., that they do not contradict each other? In this paper we formulate this and other basic problems in multi-view modeling within an abstract formal framework. We then instantiate this framework in a discrete, finite-state system setting, and study how some key verification and synthesis problems can be solved in that setting.
\end{abstract}

\section{Introduction}

Real systems are usually complex objects, and grasping all the details of a system at the same time is often difficult. In addition, each of the various stakeholders in the system are concerned with different system aspects. For these reasons, modeling and design teams usually deal only with partial and incomplete views of a system, which are easier to manage separately. For example, when designing a digital circuit, architects may be concerned with general (boolean) functionality issues, while ignoring performance. Other stakeholders, however, may be concerned about timing aspects such as the delay of the critical path, which ultimately affects the clock rate at which the circuit can be run. Yet other stakeholders may be interested in a different aspect, namely, energy consumption of the circuit which affects battery life.

Modeling and simulation are often used to support system design. In this paper, when we talk about views, we refer concretely to the different models of a system that designers build. Such models may be useful as models of an existing system: the system exists, and a model is built in order to study the system. Then, the model is only a partial or incomplete view of the system, since it focuses on certain aspects and omits others. For example, an energy consumption model for an airplane ignores control, air dynamics, and other aspects. Models may also be used for a system-to-be-built: an energy consumption model as in the example above could be developed as part of the design process, even before the airplane is built.

\footnotetext{
* This research is partially supported by the National Science Foundation and the Academy of Finland, via projects ExCAPE: Expeditions in Computer Augmented Program Engineering and COSMOI: Compositional System Modeling with Interfaces, by the Deutsche Forschungsgemeinschaft as part of the Transregional Collaborative Research Centre SFB/TR 14 AVACS, and by the centers TerraSwarm and iCyPhy (Industrial Cyber-Physical Systems) at UC Berkeley.
} 
For large systems, each aspect of the system is typically designed by a dedicated design team. These teams often use different modeling languages and tools to capture different views, which is generally referred to as multi-view modeling (MVM). MVM presents a number of challenges, such as the crucial issue of consistency: if different views of the system are captured by different models, and these models have some degree of overlap, how can we guarantee that the models are consistent, i.e., that they do not contradict each other? Understanding the precise meaning of such questions, and developing techniques to answer them, ideally fully automatically, is the main goal of this paper.

Toward this goal, we begin in Section 2 by introducing an example of simple 3dimensional structure modeling. Even though our focus is on dynamic behaviors, we will use this static system as an illustrative running example to demonstrate the salient concepts of our formal MVM framework. The latter is itself presented in Section 3. The main concepts are as follows: (1) views can be derived from systems using abstraction functions, which map system behaviors to view behaviors; (2) conformance formalizes how "faithful" a view is to a system; (3) consistency of a set of views is defined as existence of a witness system to which all views conform; (4) view reduction allows to "optimize" views by using the information contained in other views; (5) orthogonality captures independence between views.

The framework proposed in Section 3 is abstract, in the sense that it does not refer to specific notions of behaviors, neither to concrete representations of systems and views. In the rest of the paper we instantiate this abstract framework for the case of discrete systems. The latter, defined in Section 4, are finite-state symbolic transition systems consisting of a set of state variables, a predicate over the state variables characterizing the set of initial states, and a predicate characterizing the transition relation.

In Section 5 we study projections as abstraction functions for discrete systems. Fullyobservable systems, where all variables are observable, are not closed under projection, therefore we also consider systems with internal (unobservable) variables. We show how to effectively solve a number of verification and synthesis problems on discrete systems and views, including view conformance and consistency checking.

\section{Running Example: 3D Objects}

To illustrate the concept of views we introduce a running example. Consider the 3D structure shown at the left of Figure 1. It can be modeled as a set of points in a $4 \times 4 \times 4$ space, each point $(x, y, z)$ representing a "box" appearing at coordinate $(x, y, z)$, for $x, y, z \in\{1,2,3,4\}$. The object shown to the left of the figure contains 16 such boxes, and the corresponding set contains 16 points.

Three views of the object are shown to the right of the figure: a top view, a front view, and a side view. These views can be formalized as 2D projections. Let $S$ be the set of points representing the 3D object. Then the three views can be formalized as sets $V_{\text {top }}, V_{\text {front }}, V_{\text {side }}$, where: $V_{\text {top }}=\{(x, y) \mid \exists z:(x, y, z) \in S\}, V_{\text {front }}=\{(x, z) \mid \exists y:$ $(x, y, z) \in S\}, V_{\text {side }}=\{(y, z) \mid \exists x:(x, y, z) \in S\}$.

The above projections can be seen as abstractions of $S$. In fact, they are generally strict abstractions in the sense that some information about $S$ is lost during the abstraction. In the case of Figure 1, e.g., the same views would be obtained if one were to add to the object the missing boxes so that no box under the "staircase structure" hangs in the air. 

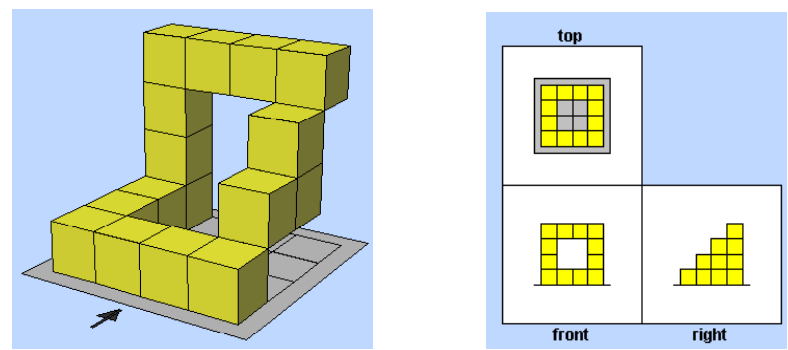

Fig. 1. A 3D structure (left) and 3 views of it (right) - image produced using this tool: http : //www.fi.uu.nl/toepassingen/02015/toepassing_wisweb.en.html.

\section{Views: a Formalization}

Systems: We define a system semantically, as a set of behaviors. As in [15], there is no restriction on the type of behaviors: they could be discrete traces, continuous trajectories, hybrid traces, or something else. We only assume given a domain of possible behaviors, $\mathcal{U}$. Then, a system $S$ over domain of behaviors $\mathcal{U}$ is a subset of $\mathcal{U}: S \subseteq \mathcal{U}$.

View domains: A view is intuitively an "incomplete picture" of a system. It can be incomplete in different ways:

- Some behaviors may be missing from the view, i.e., the view may contain only a subset of system behaviors. (As we shall see when we discuss conformance, the view may also be a superset.)

- Some parts of a behavior itself may be missing in the view. E.g., if the behavior refers to a state vector with, say, 10 state variables, the view could refer only to 2 state variables. In this case the view can be seen as a projection.

- More generally, the view may be obtained by some other kind of transformation (not necessarily a projection) to behaviors. E.g., the original system behaviors may contain temperature as a state variable, but the view only contains temperature averages over some period of time.

From the above discussion, it appears that: semantically, views can be formalized as sets of behaviors, just like systems are. However, because of projections or other transformations, the domain of behaviors of a view is not necessarily the same as the domain of system behaviors, $\mathcal{U}$. Therefore, we let $\mathcal{D}_{i}$ be the domain of behaviors of view $i$ (there can be more than one view, hence the subscript $i$ ). When we refer to a general view domain, we drop the subscript and simply write $\mathcal{D}$.

In the case of our running example, $\mathcal{U}=\{1,2,3,4\}^{3}$, and $\mathcal{D}_{\text {top }}=\mathcal{D}_{\text {front }}=$ $\mathcal{D}_{\text {side }}=\{1,2,3,4\}^{2}$.

Views: A view is a set of behaviors over a given view domain. That is, a view $V$ over view domain $\mathcal{D}$ is defined to be a subset of $\mathcal{D}: V \subseteq \mathcal{D}$.

Abstraction functions: Given a domain of behaviors $\mathcal{U}$ and a view domain $\mathcal{D}$, we would like to relate systems over $\mathcal{U}$ and views over $\mathcal{D}$. In order to do this, we will first introduce abstraction functions, which map behaviors from $\mathcal{U}$ to $\mathcal{D}$. An abstraction function from $\mathcal{U}$ to $\mathcal{D}$ is defined to be a mapping $a: \mathcal{U} \rightarrow \mathcal{D}$. Abstraction functions can be projections or other types of transformations, as discussed above. 
In the case of our running example, the abstraction functions $a_{\text {top }}, a_{\text {front }}, a_{\text {side }}$ are 3D-to-2D projections on the corresponding planes.

An abstraction function $a$ can be naturally "lifted" from behaviors to systems. If $S \subseteq \mathcal{U}$, then $a(S)$ is defined to be: $a(S):=\{a(\sigma) \mid \sigma \in S\}$. Note that $a(S) \subseteq \mathcal{D}$, therefore, $a(S)$ is a view over $\mathcal{D}$.

Conformance: Given system $S \subseteq \mathcal{U}$, view $V \subseteq \mathcal{D}$, and abstraction function $a: \mathcal{U} \rightarrow$ $\mathcal{D}$, we say that $V$ is a complete view of $S$ w.r.t. $a$ if $V=a(S)$. The notion of complete view is a reasonable way of capturing how "faithful" a given view is to a certain system. For example, if $S$ is an object containing two boxes, $S=\{(1,1,1),(2,2,2)\}$ and $a_{\text {top }}$ is the top view, then $V_{1}=\{(1,1),(2,2)\}$ is complete w.r.t. $a_{t o p}$, whereas $V_{2}=\{(2,2)\}$ and $V_{3}=\{(1,1),(2,2),(3,3)\}$ are not complete.

But faithfulness need not always require a strict equality as in the condition $V=$ $a(S)$. Depending on the usage one makes of a view, weaker conditions may be appropriate. Because of this, we introduce the notion of conformance. Conformance is defined with respect to a partial order $\sqsupseteq$ on the set of all views over view domain $\mathcal{D}$. That is, $\sqsupseteq$ is a partial order on $2^{\mathcal{D}}$, the powerset of $\mathcal{D}$. Then, we say that $V$ conforms to $S$ w.r.t. a and $\sqsupseteq$, denoted $V \sqsupseteq_{a} S$, if $V \sqsupseteq a(S)$.

For example, if one uses the top view to decide whether it is safe to drop a box to the floor without touching another box during landing, then a view that safely approximates the set of free $(x, y)$ positions could be acceptable. In this case, the partial order $\sqsupseteq$ is $\supseteq$, i.e., conformance is defined as $V \supseteq a_{t o p}(S)$. Indeed, dropping a box to $(x, y) \notin V$ would be safe, since $(x, y) \notin V$ and $V \supseteq a_{t o p}(S)$ imply $(x, y) \notin a_{t o p}(S)$. In another scenario, it may be more appropriate to require that the view under-approximates $a(S)$, thus over-approximates the set of free $(x, y)$ positions. For example, if one uses the top view to decide whether it is safe to drop an object so that it does not hit the floor, then it is more appropriate to define conformance as $V \subseteq a_{\text {top }}(S)$. In this case, $\sqsupseteq$ is $\subseteq$.

An alternative formalization - starting with conformance: In the way we formalized things so far, we started with an abstraction function $a$ and a partial order $\sqsupseteq$, and defined the conformance relation $\sqsupseteq_{a}$ with respect to those. As an alternative, we can start with a conformance relation $=\subseteq 2^{\mathcal{D}} \times 2^{\mathcal{U}}$, which relates a view $V$ and a system $S$, i.e., $V \models S$, and derive an abstraction function $a$. We can do this provided that $\models$ satisfies the conditions described below, and that the domain of views equipped with $\sqsupseteq$, denoted $\left(2^{\mathcal{D}}, \sqsupseteq\right)$, forms a complete lattice. Let $\prod$ denote the greatest lower bound in this lattice. Note that the interpretation of the lattice is that the smaller an element the more accurate it is, and $x \sqsupseteq y$ says that $y$ is smaller than $x$. Therefore, when $\sqsupseteq$ is $\supseteq$, top is $\mathcal{D}$, bottom is $\emptyset$, and $\Pi$ is $\bigcap$. When $\sqsupseteq$ is $\subseteq, \Pi$ is $\bigcup$. Then, $\models$ induces an abstraction function $a$ defined as follows:

$$
a_{\models}(S):=\prod\{V \subseteq \mathcal{D} \mid V \models S\} .
$$

For this to work, however, we need $\mid=$ to have the two following properties:

1. (monotonicity) $V_{1} \models S \wedge V_{2} \sqsupseteq V_{1} \Rightarrow V_{2} \models S$.

2. (conformance preserved by $\left.\prod\right) \forall W \subseteq 2^{\mathcal{D}}:(\forall V \in W: V \models S) \Rightarrow\left(\prod W\right) \models S$.

Condition 1 says that if $V_{1}$ conforms to $S$ then any view greater than $V_{1}$ also conforms to $S$. Condition 2 says that if a set of views all conform to a system $S$, then their greatest 
lower bound also conforms to $S$. Any relation $\sqsupseteq_{a}$ defined by an abstraction function $a$ and an order $\sqsupseteq$ forming a complete lattice has these two properties by construction.

Consistency: Consider a set of views, $V_{1}, V_{2}, \ldots, V_{n}$, over view domains $\mathcal{D}_{1}, \mathcal{D}_{2}, \ldots, \mathcal{D}_{n}$. For each view domain $\mathcal{D}_{i}$, consider given a conformance relation $\models_{i}$ (which could be derived from given abstraction function $a_{i}$ and partial order $\sqsupseteq_{i}$, or defined as a primitive notion as explained above). We say that $V_{1}, V_{2}, \ldots, V_{n}$ are consistent w.r.t. $\models_{1}, \models{ }_{2}, \ldots, \models{ }_{n}$ if there exists a system $S$ over $\mathcal{U}$ such that $\forall i=1, \ldots, n: V_{i} \models_{i} S$. We call such a system $S$ a witness to the consistency of $V_{1}, V_{2}, \ldots, V_{n}$. Clearly, if no such $S$ exists, then one must conclude that the views are inconsistent, as there is no system from which these views could be derived. When $\beth_{i}$ is $=$ for all $i$, i.e., when $V_{i}=a_{i}(S)$ for all $i$, we say that $V_{1}, \ldots, V_{n}$ are strictly consistent. Note that if $\beth_{i}$ is $\supseteq$ for all $i$, then consistency trivially holds as the empty system is a witness, since $V_{i} \supseteq \emptyset=a_{i}(\emptyset)$ for all $i$. Also, if $\sqsupseteq_{i}$ is $\subseteq$ for all $i$ and every $a_{i}$ satisfies $a_{i}(\mathcal{U})=\mathcal{D}_{i}$, then consistency trivially holds as the system $\mathcal{U}$ is a witness, since $V_{i} \subseteq \mathcal{D}_{i}=a_{i}(\mathcal{U})$ for all $i$.

In our 3D objects example, if $V_{\text {top }}$ is non-empty but $V_{\text {side }}$ is empty, then the two views are inconsistent w.r.t. strict conformance $V=a(S)$. A less trivial case is when $V_{\text {top }}=\{(1,1)\}$ and $V_{\text {side }}=\{(2,2)\}$. Again the two views are inconsistent (w.r.t. =): $V_{\text {top }}$ asserts that some box must be in the column with $(x, y)$ coordinates $(1,1)$, but $V_{\text {side }}$ implies that there is no box whose $y$ coordinate is 1 .

The last example may mislead to believe that consistency (w.r.t. $=$ ) is equivalent to "intersection of inverse projection of views being non-empty." This is not true. Even in the case where abstraction functions are projections, non-empty intersection of inverse projections is a necessary, but not a sufficient condition for consistency. To see this, consider views $V_{\text {top }}=\{(1,1),(3,3)\}$ and $V_{\text {side }}=\{(2,2),(1,2)\}$ in the context of our running example. These two views are inconsistent w.r.t. $=$. Yet the intersection of their inverse projections is non-empty, and equal to $\{(1,1,2)\}$.

View reduction: Given a set of views $V_{1}, \ldots, V_{n}$ of a system $S$, it may be possible to "reduce" each view $V_{i}$ based on the information contained in the other views, and as a result obtain views $V_{1}^{\prime}, \ldots, V_{n}^{\prime}$ that are "more accurate" views of $S$. We use the term reduction inspired from similar work in abstract interpretation $[5,10]$.

For example, if we assume that conformance is defined as $V \supseteq a(S)$, then the views $V_{\text {top }}=\{(1,1),(3,3)\}$ and $V_{\text {side }}=\{(2,2),(1,2)\}$ can be reduced to $V_{\text {top }}^{\prime}=\{(1,1)\}$ and $V_{\text {side }}^{\prime}=\{(1,2)\} . V_{\text {top }}^{\prime}$ is still a valid top view, in the sense that for every system $S$, if both $V_{\text {top }} \supseteq a_{\text {top }}(S)$ and $V_{\text {side }} \supseteq a_{\text {side }}(S)$, then $V_{\text {top }}^{\prime} \supseteq a_{\text {top }}(S)$. In addition, $V_{\text {top }}^{\prime}$ is more accurate than $V_{\text {top }}$ in the sense that $V_{t o p}^{\prime}$ is a strict subset of $V_{\text {top }}$. Indeed, $V_{\text {top }}$ does not contain the "bogus" square $(3,3)$ which cannot occur in $S$, as we learn from $V_{\text {side }}$.

Let us now define the notion of view reduction formally. First, given a conformance relation between views and systems, $=\subseteq \subseteq 2^{\mathcal{D}} \times 2^{\mathcal{U}}$, we define the concretization function $c_{\models}$ which, given a view $V$, returns the set of all systems which $V$ conforms to:

$$
c_{\models}(V):=\{S \subseteq \mathcal{U} \mid V \models S\}=\left\{S \subseteq \mathcal{U} \mid V \sqsupseteq a_{\models}(S)\right\} .
$$

Note that $V_{1}, \ldots, V_{n}$ are consistent w.r.t. $\models_{1}, \ldots, \models_{n}$ iff $\bigcap_{i=1}^{n} c_{\models_{i}}\left(V_{i}\right) \neq \emptyset$. Also observe that, by definition, $a_{\models}(S) \models S$. As a consequence, $S \in c_{\models}\left(a_{\models}(S)\right)$ for all $S \subseteq \mathcal{U}$. 
We next lift $a_{\models}$ to sets of systems. For this, we will again assume that $\left(2^{\mathcal{D}}, \sqsupseteq\right)$ forms a lattice, with $\Pi$ denoting its greatest lower bound. ${ }^{3}$ Then, if $\mathcal{S}$ is a set of systems over $\mathcal{U}$, we define $a_{\models}(\mathcal{S})$ to be the "most accurate" view that conforms to all systems in $\mathcal{S}$ :

$$
a_{\models}(\mathcal{S}):=\prod\left\{V \subseteq \mathcal{D} \mid c_{\models}(V) \supseteq \mathcal{S}\right\}=\prod\{V \subseteq \mathcal{D} \mid \forall S \in \mathcal{S}: V \models S\} .
$$

Lemma 1. The most accurate view that conforms to a set of systems $\mathcal{S}$ can also be determined from the individual systems' abstractions:

$$
a_{\models}(\mathcal{S})=\bigsqcup\left\{a_{\models}(S) \mid S \in \mathcal{S}\right\} .
$$

Missing proofs to lemmas and theorems can be found in the technical report [17].

Given the above, and assuming $n$ view domains with corresponding conformance relations, $\left(\mathcal{D}_{1}, \models_{1}\right), \ldots,\left(\mathcal{D}_{n}, \models_{n}\right)$, view reduction can be defined as follows:

$$
\text { reduce }_{i}\left(V_{1}, V_{2}, \ldots, V_{n}\right):=a_{\models_{i}}\left(\bigcap_{i=1}^{n} c_{\models_{i}}\left(V_{i}\right)\right) \text {. }
$$

Lemma 2. Reduction is a reductive operation, i.e., $V_{i} \sqsupseteq$ reduce $_{i}\left(V_{1}, V_{2}, \ldots, V_{n}\right)$ for all $i$. The set of witnesses to the consistency of views $V_{1}, \ldots, V_{n}$ is invariant under reduction, i.e., $\bigcap_{i=1}^{n} c_{\models_{i}}\left(\operatorname{reduce}_{i}\left(V_{1}, V_{2}, \ldots, V_{n}\right)\right)=\bigcap_{i=1}^{n} c_{\models_{i}}\left(V_{i}\right)$ for all $i$.

The second part of the lemma implies that reduction is idempotent, i.e., for all $i$ : reduce $_{i}\left(V_{1}, \ldots, V_{n}\right)=$ reduce $_{i}\left(V_{1}^{\prime}, \ldots, V_{n}^{\prime}\right)$, where $V_{i}^{\prime}=\operatorname{reduce}_{i}\left(V_{1}, V_{2}, \ldots, V_{n}\right)$.

Orthogonality: In some fortunate cases different aspects of a system are independent of each other. Intuitively, what this means is that each aspect can be defined separately without the need for communication between development teams to avoid inconsistencies.

Formally, we say that view domains $\mathcal{D}_{1}, \ldots, \mathcal{D}_{n}$ are orthogonal if all sets of nonempty views $V_{1}, \ldots, V_{n}$ from these view domains are mutually irreducible, i.e., if reduce $_{i}\left(V_{1}, \ldots, V_{n}\right)=V_{i}$ for all $i=1, \ldots, n$. The view domains from our example of $3 \mathrm{D}$ objects, capturing projections onto two dimensions, are not orthogonal, as the reduction example involving the domains shows. On the other hand, view domains corresponding to the projection onto individual dimensions would indeed be orthogonal to each other.

Alternatively, orthogonal view domains can be defined by requiring that all sets of non-empty views $V_{1}, \ldots, V_{n}$ from these domains are consistent w.r.t. $=$.

The following lemma shows that the two definitions of orthogonal domains are equivalent, if we assume that conformance is defined based on abstraction functions and the superset and equality relations as the partial orders on views.

Lemma 3. Given non-empty views $V_{1}, \ldots, V_{n}$, the following statements are equivalent:

1. $V_{1}, \ldots, V_{n}$ are consistent w.r.t. $={ }_{a_{1}}, \ldots,=a_{n}$.

2. $V_{1}, \ldots, V_{n}$ are mutually irreducible w.r.t. $\supseteq_{a_{1}}, \ldots, \supseteq_{a_{n}}$.

3. $V_{1}, \ldots, V_{n}$ are mutually irreducible w.r.t. $\subseteq_{a_{1}}, \ldots, \subseteq_{a_{n}}$.

\footnotetext{
${ }^{3}$ Note that when $\sqsupseteq$ is a set-theoretic relation such as $\subseteq$ or $\supseteq$, this obviously holds and $\prod$ is $\bigcup$ or $\bigcap$. When $\sqsupseteq$ is $=$ then $\left(2^{\mathcal{D}},=\right)$ is not a lattice, and the definition of view reduction given below does not apply. This is not a problem, as in that case we require views to be complete.
} 
A system $S \subseteq \mathcal{U}$ is called view definable w.r.t. $\models_{1}, \ldots, \models_{n}$ if there exist views $V_{1} \subseteq \mathcal{D}_{1}, \ldots, V_{n} \subseteq \mathcal{D}_{n}$, such that $c_{\models_{1}}\left(V_{1}\right) \cap \cdots \cap c_{\models}{ }_{n}\left(V_{n}\right)=\{S\}$. In the example of 3D objects, with 2D projections, the empty object $S^{n}=\{\}$ is view definable, as it is defined by the empty views. Similarly, all objects $S_{i, j, k}=\{(i, j, k)\}$ are view definable. Note that a general cube is not view definable, as there are other objects (e.g., a hollow cube) which have the same $2 \mathrm{D}$ projections.

\section{Verification and synthesis problems related to views:}

View conformance checking: given (concrete representation of) system $S$, view $V$, and a certain conformance relation, does $V$ conform to $S$ ?

View synthesis: given system $S$ and abstraction function $a$, synthesize (concrete representation of) $a(S)$. Alternatively, given $S$ and conformance relation $\models$, construct smallest view $V$ such that $V \mid=S$, that is, construct $a_{\models}(S)$.

View consistency checking: given views $V_{1}, \ldots, V_{n}$ and conformance relations $\models_{1}, \ldots, \models_{n}$, check whether $V_{1}, \ldots, V_{n}$ are consistent w.r.t. $\models_{1}, \ldots, \models_{n}$.

System synthesis from views: given consistent views $V_{1}, \ldots, V_{n}$ and conformance relations $=_{1}, \ldots,=_{n}$, construct a system $S$ such that for all $i, V_{i} \models_{i} S$.

View reduction: given views $V_{1}, \ldots, V_{n}$ compute reduce $_{i}\left(V_{1}, V_{2}, \ldots, V_{n}\right)$ for given $i$.

\section{Discrete Systems}

Our goal in the rest of this paper is to instantiate the view framework developed in Section 3. We instantiate it for a class of discrete systems, and we also provide answers to some of the corresponding algorithmic problems.

We will consider finite-state discrete systems. The state space of such a system can be represented by a set of boolean variables, $X$, resulting in $2^{n}$ potential states, where $n=|X|$ is the size of $X$. A state $s$ over $X$ is a valuation over $X$, i.e., a function $s: X \rightarrow \mathbb{B}$, where $\mathbb{B}:=\{0,1\}$ is the set of booleans. For convenience, we sometimes consider other finite domains with the understanding that they can be encoded as booleans. A behavior over $X$ is a finite or infinite sequence of states over $X, \sigma=s_{0} s_{1} s_{2} \cdots$. $\mathcal{U}(X)$ denotes the set of all possible behaviors over $X$.

Semantically, a discrete system $S$ over $X$ is a set of behaviors over $X$, i.e., $S \subseteq$ $\mathcal{U}(X)$. For computation, we need a concrete representation of discrete systems. We will start with a simple representation where all system variables are observable. We will then discuss limitations of this representation and consider an extension where the system can also have internal (unobservable) variables in addition to the observable ones.

Fully-observable discrete systems: A fully-observable discrete system (FOS) is represented concretely by a triple $(X, \theta, \phi) . X$ is the (finite) set of (boolean) variables. All variables in $X$ are considered observable. $\theta$ is a boolean expression over $X$, characterizing the set of initial states of the system. Given state $s$, we write $\theta(s)$ to denote the fact that $s$ satisfies $\theta$, i.e., $s$ is an initial state. $\phi$ is a boolean expression over $X \cup X^{\prime}$, where $X^{\prime}$ is the set of primed copies of variables in $X, X^{\prime}:=\left\{x^{\prime} \mid x \in X\right\}$, representing the next state variables, as usual. $\phi$ characterizes pairs of states $\left(s, s^{\prime}\right)$, each representing a transition of $S$, i.e., a move from state $s$ to state $s^{\prime}$. We write $\phi\left(s, s^{\prime}\right)$ to denote that the pair $\left(s, s^{\prime}\right)$ satisfies $\phi$, i.e., that there is a transition from $s$ to $s^{\prime}$. 
A behavior of a system $(X, \theta, \phi)$ is a finite or infinite sequence of states over $X$, $\sigma=s_{0} s_{1} s_{2} \cdots$, such that $\theta\left(s_{0}\right)$ and $\forall i: \phi\left(s_{i}, s_{i+1}\right)$, i.e., $s_{0}$ is an initial state and there is a transition from each $s_{i}$ to $s_{i+1}$ (if the latter exists). A state $s$ is reachable if there is a finite behavior $s_{0} s_{1} \cdots s_{n}$, such that $s=s_{n}$.

We sometimes use $S=(X, \theta, \phi)$ to denote the concrete (syntactic) representation of discrete system $S$, and $\llbracket S \rrbracket$ to denote its semantics, i.e., its set of behaviors.

Projection (variable hiding): Projection, or variable hiding, is a natural operation on systems, which can also serve as a basic abstraction function for views, as we shall see below. Here, we define projection and motivate the introduction of internal variables in the concrete representation of discrete systems.

Let $s$ be a state over a set of variables $X$. Given subset $Y \subseteq X$, the projection function $h_{Y}$ projects $s$ onto the set of variables $Y$, that is, $h_{Y}$ hides from $s$ all variables in $X \backslash Y . h_{Y}(s)$ is defined to be the new state $s^{\prime}$ over $Y$, that is, the function $s^{\prime}: Y \rightarrow \mathbb{B}$, such that $s^{\prime}(x)=s(x)$ for all $x \in Y$.

Projection can be lifted to behaviors in the standard way. If $\sigma=s_{0} s_{1} \cdots$ is a behavior over $X$, then $h_{Y}(\sigma)$ is a behavior over $Y$ defined by $h_{Y}(\sigma):=h_{Y}\left(s_{0}\right) h_{Y}\left(s_{1}\right) \cdots$. Projection can also be lifted to systems. If $S$ is a discrete system over $X$ then $h_{Y}(\llbracket S \rrbracket):=$ $\left\{h_{Y}(\sigma) \mid \sigma \in \llbracket S \rrbracket\right\}$.

\section{Non-closure properties}

Non-closure under projection: The projection $h_{Y}(\llbracket S \rrbracket)$ is defined semantically, as a set of behaviors. It is natural to ask whether the syntactic representation of discrete systems is closed under projection. That is, is it true that for any $S=(X, \theta, \phi)$, and $Y \subseteq X$, there exists $S^{\prime}=\left(Y, \theta^{\prime}, \phi^{\prime}\right)$, such that $\llbracket S^{\prime} \rrbracket=h_{Y}(\llbracket S \rrbracket)$ ? This is not generally true:

Lemma 4. There exists a FOS $S=(X, \theta, \phi)$, and $Y \subseteq X$, such that there is no FOS $S^{\prime}=\left(Y, \theta^{\prime}, \phi^{\prime}\right)$, such that $\llbracket S^{\prime} \rrbracket=h_{Y}(\llbracket S \rrbracket)$.

Proof. Consider the finite-state system $S=\left(\{x, y\}, x=0 \wedge y=\right.$ true, $\left(x^{\prime}=(x+\right.$ 1) $\left.\bmod 5) \wedge\left(y^{\prime} \leftrightarrow\left(x^{\prime}=0\right)\right)\right)$, where $x \in\{0,1,2,3,4\}$ and $y \in \mathbb{B}$. Let $Y=\{y\}$. Then $h_{Y}(\llbracket S \rrbracket)=\left\{y_{0} y_{1} \cdots \mid \forall i: y_{i} \Leftrightarrow i \bmod 5=0\right\}$. We claim that there is no $S^{\prime}=\left(Y, \theta^{\prime}, \phi^{\prime}\right)$ such that $\llbracket S^{\prime} \rrbracket=h_{Y}(\llbracket S \rrbracket)$. The reason is that $S^{\prime}$ needs to count modulo five in order to produce the correct output. But $S^{\prime}$ has only one boolean variable $y$.

As it turns out, we can check whether closure under projection holds for a given system: see Theorem 2 in Section 5.

Non-closure under union:

Lemma 5. Fully-observable systems over a set of variables $X$ are not closed under union, i.e., there exist $S_{1}=\left(X, \theta_{1}, \phi_{1}\right), S_{2}=\left(X, \theta_{2}, \phi_{2}\right)$ such that there is no $S=$ $(X, \theta, \phi)$ such that $\llbracket S \rrbracket=\llbracket S_{1} \rrbracket \cup \llbracket S_{2} \rrbracket$.

Proof. Consider as an example $S_{1}=\left(\{x\}, \theta_{1}=x, \phi_{1}=x \wedge \neg x^{\prime}\right)$ and $S_{2}=\left(\{x\}, \theta_{2}=\right.$ $\left.\neg x, \phi_{1}=\neg x \wedge x^{\prime}\right)$. Both systems allow exactly one transition, from $x \mapsto$ true to $x \mapsto$ false and vice versa. A system that represents the union of $S_{1}$ and $S_{2}$ needs to include both transitions. Then, however, it also includes arbitrarily long behaviors alternating between $x \mapsto$ true and $x \mapsto$ false. 
Discrete systems with internal variables: The above non-closure properties motivate us to study, in addition to fully-observable discrete systems, a generalization which extends them with a set of internal, unobservable state variables. Most practical modeling languages also allow the construction of models with both internal and observable state variables.

Accordingly, we extend the definition of a discrete system to be in general a tuple $(X, Z, \theta, \phi)$, where $X, Z$ are disjoint (finite) sets of variables. $X$ models the observable and $Z$ the internal variables. $\theta$ is a boolean expression over $X \cup Z$ and $\phi$ is a boolean expression over $X \cup Z \cup X^{\prime} \cup Z^{\prime}$. In such a system, we need to distinguish between behaviors, and observable behaviors. A behavior of a system $S=(X, Z, \theta, \phi)$ is a finite or infinite sequence $\sigma$ over $X \cup Z$, defined as above. The observable behavior corresponding to $\sigma$ is $h_{X}(\sigma)$, which is a behavior over $X$. From now on, $\llbracket S \rrbracket$ denotes the set of all behaviors (over $X \cup Z$ ) of $S$, and $\llbracket S \rrbracket_{o}$ denotes the set of observable behaviors (over $X$ ) of $S$.

Note that we allow $Z$ to be empty. In that case, the system has no internal variables, i.e., it is a FOS. We will continue to represent a FOS by a triple $S=(X, \theta, \phi)$. A FOS $S$ satisfies $\llbracket S \rrbracket=\llbracket S \rrbracket_{o}$.

Closure properties: We have already shown (Lemma 5) that FOS are not closed under union. They are however closed under intersection:

Lemma 6. Given two FOS $S_{1}=\left(X, \theta_{1}, \phi_{1}\right)$ and $S_{2}=\left(X, \theta_{2}, \phi_{2}\right)$, a FOS $S$ such that $\llbracket S \rrbracket=\llbracket S_{1} \rrbracket \cap \llbracket S_{2} \rrbracket$ is $S_{1} \wedge S_{2}=\left(X, \theta_{1} \wedge \theta_{2}, \phi_{1} \wedge \phi_{2}\right)$.

General discrete systems (with internal variables) are closed under intersection, union, as well as projection.

Lemma 7. Let $S_{1}=\left(X, Z_{1}, \theta_{1}, \phi_{1}\right)$ and $S_{2}=\left(X, Z_{2}, \theta_{2}, \phi_{2}\right)$ be two systems, such that $Z_{1} \cap Z_{2}=\emptyset$. Let $Y \subseteq X$ and let $z$ be a fresh variable not in $X \cup Z_{1} \cup Z_{2}$. Let:

$$
\begin{aligned}
& S_{\cap}=\left(X, Z_{1} \cup Z_{2}, \theta_{1} \wedge \theta_{2}, \phi_{1} \wedge \phi_{2}\right), \\
& S_{\cup}=\left(X, Z_{1} \cup Z_{2} \cup\{z\},\left(\theta_{1} \wedge z\right) \vee\left(\theta_{2} \wedge \neg z\right) .\left(z \rightarrow \phi_{1} \wedge z^{\prime}\right) \wedge\left(\neg z \rightarrow \phi_{2} \wedge \neg z^{\prime}\right)\right), \\
& S_{h}=\left(Y, Z_{1} \cup(X \backslash Y), \theta_{1}, \phi_{1}\right) .
\end{aligned}
$$

Then, $\llbracket S_{\cap} \rrbracket_{o}=\llbracket S_{1} \rrbracket_{o} \cap \llbracket S_{2} \rrbracket_{o}, \llbracket S_{\cup} \rrbracket_{o}=\llbracket S_{1} \rrbracket_{o} \cup \llbracket S_{2} \rrbracket_{o}$, and $\llbracket S_{h} \rrbracket_{o}=h_{Y}\left(\llbracket S_{1} \rrbracket_{o}\right)$.

\section{Views of Finite-State Discrete Systems}

Having defined discrete systems, we now turn to instantiating the view framework for such systems.

Discrete views, view domains, and abstraction functions: Discrete views are finitestate discrete systems. They are represented in general by tuples of the form $(X, Z, \theta, \phi)$, and when $Z=\emptyset$, by triples of the form $(X, \theta, \phi)$.

In this paper, we will study projection as the abstraction function for the discrete view framework. That is, a system will be a discrete system $S$ over a set of observable variables $X$, and therefore the domain of system behaviors will be $\mathcal{U}=\mathcal{U}(X)$. A view 
will be a discrete system $V$ over a subset of observable variables $Y \subseteq X$. Therefore, the view domain of $V$ is $\mathcal{D}=\mathcal{U}(Y)$. Note that both $S$ and $V$ may have (each their own) internal variables.

Let $S=(X, Z, \theta, \phi)$ be a discrete system, $V=\left(Y, W, \theta^{\prime}, \phi^{\prime}\right)$ be a discrete view, with $Y \subseteq X$, and $\sqsupseteq$ be one of the orders $=, \subseteq$, or $\supseteq$. To make notation lighter, we will write $V \sqsupseteq h_{Y}(S)$ instead of $\llbracket V \rrbracket_{o} \sqsupseteq h_{Y}(\llbracket S \rrbracket)$. Note, that $h_{Y}(\llbracket S \rrbracket)=h_{Y}\left(\llbracket S \rrbracket_{o}\right)$. More generally, when comparing systems or views, we compare them w.r.t. their observable behaviors. For instance, when writing $V_{1} \sqsupseteq V_{2}$, we mean $\llbracket V_{1} \rrbracket_{o} \sqsupseteq \llbracket V_{2} \rrbracket_{o}$.

Least and greatest fully-observable views: Let $S$ be a discrete system over set of observable variables $X$. Given a set $Y \subseteq X$, one might ask whether there is a "canonical" view $V$ of $S$ w.r.t. $Y$. Clearly, if we allow $V$ to have internal variables, the answer is yes: it suffices to turn all variables in $X \backslash Y$ into internal variables in $V$. Then, by Lemma 7, $V$ represents precisely the projection of $S$ to $Y$, i.e., it is a complete view, it satisfies $V=h_{Y}(S)$, and therefore trivially also $V \supseteq h_{Y}(S)$ and $V \subseteq h_{Y}(S)$. Note that this is true independently of whether $S$ has internal variables or not.

In this section we study the question for the case where we forbid $V$ from having internal variables, i.e., we restrict views to be fully-observable. As FOS are not closed under projection, there are systems that have no complete fully-observable view. On the other hand, there can be multiple views $V$ over $Y$ such that $V \supseteq h_{Y}(S)$ or $V \subseteq h_{Y}(S)$. In particular, $(Y$, true, true $) \supseteq h_{Y}(S)$ and $(Y$, false, false $) \subseteq h_{Y}(S)$, for any $S$ and $Y$. Thus, the question arises, whether there is a least fully-observable view $l v(S, Y)$ of $S$ with $l v(S, Y) \supseteq h_{Y}(S)$, such that for any fully-observable view $V^{\prime}$ with $V^{\prime} \supseteq h_{Y}(S)$, we have $V^{\prime} \supseteq \operatorname{lv}(S, Y)$. Similarly, one may ask whether there is a greatest fullyobservable view $g v(S, Y)$ w.r.t. $\subseteq_{h_{Y}}$. These questions are closely related to whether views are closed under intersection and union. In particular, we can use closure under intersection to show that a least view always exists. A greatest view, on the other hand, does not necessarily exist.

Theorem 1. Let $S=(X, Z, \theta, \phi)$ be any discrete system and let $Y \subseteq X$. Let $\psi_{S}$ characterize the set of reachable states of $S$. Then the FOS

$$
\left(Y, \theta_{Y}=\exists(X \cup Z) \backslash Y: \theta, \phi_{Y}=\exists(X \cup Z) \backslash Y: \psi_{S} \wedge \exists\left(X^{\prime} \cup Z^{\prime}\right) \backslash Y^{\prime}: \phi\right)
$$

is the unique fully-observable least view $l v(S, Y)$, that is, $l v(S, Y) \supseteq h_{Y}(S)$, and for any fully-observable view $V^{\prime}$ over $Y$ with $V^{\prime} \supseteq h_{Y}(S)$, we have $V^{\prime} \supseteq l v(S, Y)$.

As Lemma 4 shows, the projection of a system cannot generally be represented as a fully-observable view. As it turns out, we can effectively check whether this is the case for a given system $S$, by checking whether the least view of $S$ conforms to $S$ w.r.t. =.

Theorem 2. Given discrete system $S$ over $X$ and $Y \subseteq X$, there exists a fully-observable view $V$ over $Y$ with $V=h_{Y}(S)$ iff $\llbracket l v(S, Y) \rrbracket=h_{Y}(\llbracket S \rrbracket)$.

Theorem 2 implies that it is decidable to check whether a system admits a fullyobservable complete view $V$. 
Theorem 3. There is a discrete system $S$ over $X$ and a subset $Y \subseteq X$ for which there is no unique greatest fully-observable view $g v(S, Y)$ with $g v(S, Y) \subseteq h_{Y}(S)$, such that for any fully-observable view $V^{\prime}$ with $V^{\prime} \subseteq h_{Y}(S)$, we have $V^{\prime} \subseteq g v(S, Y)$.

Proof. Consider the FOS $S=\left(\{x, y\}, \theta=(x \wedge y) \vee(\neg x \wedge \neg y), \phi=\left(x \wedge \neg x^{\prime} \wedge y \wedge\right.\right.$ $\left.\left.y^{\prime}\right) \vee\left(\neg x \wedge x^{\prime} \wedge \neg y \wedge \neg y^{\prime}\right)\right)$. The FOS $S_{1}$ and $S_{2}$ from the proof of Lemma 5 are both views of $S$ for $Y=\{x\}$, yet they are incomparable and there is no FOS view conforming to $S$ w.r.t. $\subseteq$ that is greater than both of them as their union is not a view of $S$.

\section{View conformance checking for discrete systems and views:}

Problem 1. Given discrete system $S=(X, Z, \theta, \phi)$, discrete view $V=\left(Y, W, \theta_{V}, \phi_{V}\right)$, where $Y \subseteq X$ and $Z \cap W=\emptyset$, and partial order $\sqsupseteq \in\{=, \subseteq, \supseteq\}$, check whether $V \sqsupseteq h_{Y}(S)$.

Problem 2. Given discrete systems $S_{1}=\left(X, Z_{1}, \theta_{1}, \phi_{1}\right)$ and $S_{2}=\left(X, Z_{2}, \theta_{2}, \phi_{2}\right)$, where $Z_{1} \cap Z_{2}=\emptyset$, and partial order $\sqsupseteq \in\{=, \subseteq, \supseteq\}$, check whether $\llbracket S_{1} \rrbracket_{o} \sqsupseteq \llbracket S_{2} \rrbracket_{o}$.

Theorem 4. Problem 1 can be reduced to Problem 2 in polynomial time. Problem 2 is in PSPACE.

Proof. For the first part of the theorem, observe that discrete systems are closed under projection. An instance of Problem 1 can be transformed into an instance of Problem 2, simply by shifting the variables $X \backslash Y$ of $S$ from the observable to the internal variables.

For the second part of the theorem, we limit our attention to the case $\sqsupseteq=\subseteq$, as the other two cases then follow trivially. Problem 2 can be reduced to the finite state automaton inequivalence problem, which is known to be in PSPACE [9]. As discrete systems are closed under union, we construct a system $S_{\cup}$, with $\llbracket S_{\cup} \rrbracket_{o}=\llbracket S_{1} \rrbracket_{o} \cup \llbracket S_{2} \rrbracket_{o}$. Then $\llbracket S_{\cup} \rrbracket_{o}=\llbracket S_{2} \rrbracket_{o}$ iff $\llbracket S_{1} \rrbracket_{o} \subseteq \llbracket S_{2} \rrbracket_{o}$. From $S_{\cup}$ and $S_{2}$ we can construct NFAs $M_{\cup}$ and $M_{2}$ that accept a sequence $\sigma$ iff $\sigma$ is an observable behavior of $S_{\cup}$ and $S_{2}$, respectively.

Theorem 5. Problem 1 is in $P$ for partial order $\sqsupseteq=\supseteq$ if the discrete view $V$ is a FOS.

Proof. First, notice that if $Y \subseteq X$, then $V=\left(Y, \theta_{V}, \phi_{V}\right)$ is a view of $S=(X, Z, \theta, \phi)$ if and only if it is a view of the fully-observable system $S^{\prime}=(X \cup Z, \theta, \phi)$. This is because $h_{Y}(S)=h_{Y}\left(S^{\prime}\right)$. Thus, in the following, we will assume $S$ to be a FOS with $S=(X, \theta, \phi)$.

Let $\psi_{S}$ denote the reachable states of $S . \psi_{S}$ can, e.g., be computed incrementally using BDDs. Let $Z:=X \backslash Y$ and $Z^{\prime}:=X^{\prime} \backslash Y^{\prime}$. Then, $V \supseteq_{h_{Y}} S$, if and only if the following two conditions hold, which can be effectively checked:

1. $\forall Y, Z: \theta(Y, Z) \rightarrow \theta_{V}(Y) \equiv \forall s: \theta(s) \rightarrow \theta_{V}\left(h_{Y}(s)\right)$, and

2. $\forall Y, Z, Y^{\prime}, Z^{\prime}: \psi_{S}(Y, Z) \rightarrow\left(\phi\left((Y, Z),\left(Y^{\prime}, Z^{\prime}\right)\right) \rightarrow \phi_{V}\left(Y, Y^{\prime}\right)\right) \equiv \forall s, s^{\prime}$ : $\psi_{S}(s) \rightarrow\left(\phi\left(s, s^{\prime}\right) \rightarrow \phi_{V}\left(h_{Y}(s), h_{Y}\left(s^{\prime}\right)\right)\right)$.

We need to show that Conditions 1 and 2 from above hold, if and only if $V \supseteq h_{Y} S$. Let us first show that Conditions 1 and 2 imply $V \supseteq h_{Y} S$ :

We show this by induction over the length $n$ of behaviors $\sigma$ of $S$.

Base case: let $\sigma=s_{0} \in \llbracket S \rrbracket$ be any behavior of length 1 of $S$. Then $\theta\left(s_{0}\right)$ must hold, 
which, by Condition 1 implies $\theta_{V}\left(h\left(s_{0}\right)\right)$, which implies that $h\left(s_{0}\right) \in \llbracket V \rrbracket$.

Induction step: let $\sigma=s_{0} s_{1} \cdots s_{n-1} s_{n} \in \llbracket S \rrbracket$ be a sequence of length $n+1$. As $S$ is by definition prefix-closed, $s_{0} s_{1} \cdots s_{n-1}$ is also in $S$. By the induction hypothesis, we know that $h\left(s_{0}\right) h\left(s_{1}\right) \cdots h\left(s_{n-1}\right)$ is in $\llbracket V \rrbracket$. As $\sigma \in S, s_{n-1}$ is reachable, thus $\psi_{S}\left(s_{n-1}\right)$ holds. Thus, we can apply Condition 2 , and deduce from the fact that $\phi\left(s_{n-1}, s_{n}\right)$, that $\phi_{V}\left(h\left(s_{n-1}\right), h\left(s_{n}\right)\right)$. This in turn implies that $h\left(s_{0}\right) h\left(s_{1}\right) \cdots h\left(s_{n-1}\right) h\left(s_{n}\right)$ is a behavior of $V$.

Now, let us show the opposite direction, i.e., that $V \supseteq \bigcap_{Y} S$ implies Conditions 1 and 2. We show this by contraposition. Assume Condition 1 does not hold. Then, there is a valuation $v Y$ of $Y$ and a valuation $v Z$ of $Z$, such that $\theta(v Y v Z)$ holds (where $v Y v Z$ is the valuation that agrees with $v Y$ on $Y$ and with $v Z$ on $Z$ ), but $\theta_{V}(v Y)$ does not. Clearly, $h(v Y v Z)=v Y$. So, $v Y v Z \in \llbracket S \rrbracket$, but $h(v Y v Z) \notin$ $\llbracket V \rrbracket$, which implies that $V \supseteq_{h_{Y}} S$ does not hold. Now assume that Condition 2 does not hold. This implies that there are valuations $v Y, v Z$ and $v Y^{\prime}, v Z^{\prime}$, such that $\psi_{S}(v Y v Z)$ and $\phi\left(v Y v Z, v Y^{\prime} v Z^{\prime}\right)$ hold, but $\phi_{V}\left(v Y, v Y^{\prime}\right)$ does not. As $v Y v Z$ is thus reachable, there must be a behavior $s_{0} \cdots(v Y v Z) \in \llbracket S \rrbracket$. By $\phi\left(v Y v Z, v Y^{\prime} v Z^{\prime}\right)$, we also have that $s_{0} \cdots(v Y v Z)\left(v Y^{\prime} v Z^{\prime}\right) \in \llbracket S \rrbracket$. Yet, because $\phi_{V}\left(v Y, v Y^{\prime}\right)$ does not hold, $h\left(s_{0}\right) \cdots h(v Y v Z) h\left(v Y^{\prime} v Z^{\prime}\right) \notin \llbracket V \rrbracket$, which concludes the proof.

Theorem 6. Problem 1 is PSPACE-hard even if the discrete view $V$ is fully-observable for $|Y| \geq 1$ and partial orders $=, \subseteq$. Problem 1 is also PSPACE-hard for $|Y| \geq 1$ and partial order $\supseteq$ if $V$ is not restricted to be fully-observable.

In [13], it is shown that checking the universality of non-deterministic finite automata (NFA), having the property that all states are final, is PSPACE-hard for alphabets of size at least 2. In the technical report [17], we show how to reduce this problem to Problem 1.

\section{View consistency checking for discrete systems and views:}

Problem 3. Given partial order $\sqsupseteq \in\{=, \subseteq, \supseteq\}$ and discrete views $V_{1}, \ldots, V_{n}$, with $V_{i}=\left(Y_{i}, W_{i}, \theta_{i}, \phi_{i}\right)$ for $i=1, \ldots, n$, check whether there exists discrete system $S=(X, Z, \theta, \phi)$, with $X \supseteq Y_{i}$ for all $i$, such that $V_{i} \sqsupseteq_{h_{Y_{i}}} S$ for all $i$.

Problem 3 asks to check whether a given number of views are consistent w.r.t. projection as abstraction function and a given partial order among $=, \subseteq, \supseteq$. Note that we can assume without loss of generality that the witness system has set of observable variables $X=\bigcup_{i=1}^{n} Y_{i}$, as any extra variables could be made internal.

Problem 3 is trivially solved by the "all" system $\theta=\phi=t r u e$ for $\subseteq$ and by the "empty" system $\theta=\phi=$ false for $\supseteq$. For $=$, if we restrict the witness system to be a FOS, then Problem 3 is trivially decidable as there are only finitely many systems with $X=\bigcup_{i=1}^{n} Y_{i}$. Clearly, this is not very efficient. Theorems 7-9 (which also apply to general discrete systems, non necessarily FOS) provide a non-brute-force method.

Theorem 7. For a set of views $V_{1}, \ldots, V_{n}$, with $V_{i}=\left(Y_{i}, W_{i}, \theta_{i}, \phi_{i}\right)$ for all $i$, there always exists a computable unique greatest witness system $g w\left(V_{1}, \ldots, V_{n}\right)=(X, Z, \theta, \phi)$, with $X=\bigcup_{i=1}^{n} Y_{i}$, w.r.t. partial order $\supseteq$. 
Proof. First, observe that $S_{i}=\left(X, W_{i}, \theta_{i}, \phi_{i}\right)$ is the unique greatest witness system for $V_{i}$ for systems with the set of variables $X$, i.e., $V_{i} \supseteq_{h_{Y_{i}}} S_{i}$ and for all $S=(X, W, \theta, \phi)$ such that $V_{i} \supseteq h_{Y_{i}} S$, we have $\llbracket S_{i} \rrbracket \supseteq \llbracket S \rrbracket$. In fact, $V_{i}=_{h_{Y_{i}}} S_{i}$. Given two views $V_{i}, V_{j}$, the unique greatest witness system for both views is $S_{i, j}=\left(X, W_{i} \cup W_{j}, \theta_{i} \wedge \theta_{j}, \phi_{i} \wedge \phi_{j}\right)$, whose behaviors are exactly the intersection of the behaviors of $S_{i}$ and $S_{j}$ (see Lemma 7). Adding any behavior to $S_{i, j}$ would violate either $V_{i} \supseteq h_{Y_{i}} S_{i, j}$ or $V_{j} \supseteq_{h_{Y_{i}}} S_{i, j}$ Generalizing the above, $S_{\wedge}=\left(X_{\wedge}=\bigcup_{i=1}^{n} Y_{i}, Z_{\wedge}=\bigcup_{i=1}^{n} W_{i}, \theta_{\wedge}=\bigwedge_{i=1}^{n} \theta_{i}, \phi_{\curlywedge}=\bigwedge_{i=1}^{n} \phi_{i}\right)$ is the unique greatest witness system for the set of views $V_{1}, \ldots, V_{n}$.

Theorem 8. Consistency with respect to $=$ holds if and only if the greatest witness system $g w\left(V_{1}, \ldots, V_{n}\right)$ derived in Theorem 7 is a witness with respect to $=$.

Theorem 9. Problem 3 is PSPACE-complete for partial order $=$.

Theorem 10. There are discrete views $V_{1}, \ldots, V_{n}$, with $V_{i}=\left(Y_{i}, W_{i}, \theta_{i}, \phi_{i}\right)$ for all $i$, for which there is no unique least witness system $l w\left(V_{1}, \ldots, V_{n}\right)=(X, Z, \theta, \phi)$, with $X=\bigcup_{i=1}^{n} Y_{i}$, w.r.t. partial order $\subseteq$.

Proof. Consider the following two views $V_{x}=\left(\{x\}, \theta_{x}=x, \phi_{x}=\right.$ true $)$ and $V_{y}=$ $\left(\{y\}, \theta_{y}=y, \phi_{y}=\right.$ true $)$. We provide two witness systems $S_{1}, S_{2}$, both consistent with $V_{x}, V_{y}$, such that their intersection is not consistent with $V_{x}$ and $V_{y}$, which proves that there is no unique least witness system for $V_{x}, V_{y}$ w.r.t. $\subseteq$ :

$$
\begin{aligned}
& S_{1}=\left(\{x, y\}, \theta_{1}=x \wedge y, \phi_{1}=(x \Leftrightarrow y) \wedge\left(x^{\prime} \Leftrightarrow y^{\prime}\right)\right) \\
& S_{2}=\left(\{x, y\}, \theta_{2}=x \wedge y, \phi_{2}=x^{\prime} \vee y^{\prime}\right)
\end{aligned}
$$

In every behavior of $S_{1}, x$ and $y$ take the same value, whereas in $S_{2}, x$ and $y$ are never both false. In their intersection $S_{\cap}=\left(\{x, y\}, \theta_{1} \wedge \theta_{2}, \phi_{1} \wedge \phi_{2}\right)$, neither $x$ nor $y$ can thus ever be false. So $S_{\cap}$ is neither consistent with $V_{x}$ nor with $V_{y}$.

\section{View reduction for discrete systems and views:}

Problem 4. Given partial order $\sqsupseteq \in\{=, \subseteq, \supseteq\}$ and discrete views $V_{1}, \ldots, V_{n}$, with $V_{i}=$ $\left(Y_{i}, W_{i}, \theta_{i}, \phi_{i}\right)$ for $i=1, \ldots, n$, compute reduce $_{i}\left(V_{1}, \ldots, V_{n}\right)$ for all $i=1, \ldots, n$.

Theorem 11. For partial order $\supseteq$, Problem 4 is solved by the projection of the greatest witness system to the observable variables of the respective view: let $g w\left(V_{1}, \ldots, V_{n}\right)=$ $(X, Z, \theta, \phi)$, with $X=\bigcup_{i=1}^{n} Y_{i}$, be the greatest witness system to the consistency of $V_{1}, \ldots, V_{n}$ w.r.t. partial order $\supseteq$. Then:

$$
\text { reduce }_{i}\left(V_{1}, \ldots, V_{n}\right)=\left(Y_{i}, Z \cup\left(X \backslash Y_{i}\right), \theta, \phi\right) .
$$

For partial order $\subseteq$, Problem 4 is often trivial. Specifically, if the sets of observable variables of all views are incomparable, then no information can be transferred from one view to another:

Theorem 12. Let $V_{1}, \ldots, V_{n}$ be discrete views with $V_{i}=\left(Y_{i}, W_{i}, \theta_{i}, \phi_{i}\right)$. Assume $Y_{i} \backslash$ $Y_{j} \neq \emptyset$ for all $i, j$. Then, assuming $\sqsupseteq i s \subseteq$, the following holds for all $i$ :

$$
\text { reduce }_{i}\left(V_{1}, \ldots, V_{n}\right)=V_{i} \text {. }
$$




\section{Discussion}

MVM is not a new topic, and terms such as "view" and "viewpoint" often appear in system engineering literature, including standards such as ISO 42010 [12]. Despite this fact, and the fact that MVM is a crucial concern in system design, an accepted mathematical framework for reasoning about views has so far been lacking. This is especially true for behavioral views, that is, views describing the dynamic behavior of the system, as opposed to its static structure. Behavioral views are the main focus of our work.

Discrete behavioral views could also be captured in a temporal logic formalism such as LTL. View consistency could then be defined as satisfiability of the conjunction $\phi_{1} \wedge \cdots \wedge \phi_{n}$, where each $\phi_{i}$ is a view (possibly over a different set of variables). This definition is however weaker than our definition of strict consistency (w.r.t. $=$ ). Satisfiability of $\phi_{1} \wedge \cdots \wedge \phi_{n}$ is equivalent to checking that the intersection of the inverse projections of views is non-empty, which, as we explained earlier, is a necessary but not sufficient condition for strict consistency.

The same fundamental difference exists between our framework and view consistency as formulated in the context of interface theories, where a special type of interface conjunction is used [11] (called "fusion" in [2] and "shared refinement" in $[7,18]$ ).

Behavioral abstractions/views are also the topic of $[15,16]$. Their framework is close to ours, in the sense that it also uses abstraction functions to map behaviors between different levels of abstraction (or between systems and views). The focus of both $[15,16]$ is to ease the verification task in a heterogeneous (e.g., both discrete and continuous) setting. Our main focus is checking view consistency. The notion of "heterogeneous consistency" [15] is different from our notion of view consistency. The notion of "conjunctive implication" [15] is also different, as views which have an empty intersection of their inverse projections trivially satisfy conjunctive implication, yet these views can be inconsistent in our framework. Problems such as view consistency checking are not considered in $[15,16]$.

Consistency between architectural views, which capture structural but not behavioral aspects of a system, is studied in [3]. Consistency problems are also studied in [8] using a static, logic-based framework. Procedures such as join and normalization in relational databases also relate to notions of static consistency.

An extensive survey of different approaches for multi-view modeling can be found in [14]. [14] also gives a partial and preliminary formalization, but does not discuss algorithmic problems. [4] discusses an informal methodology for selecting formalisms, languages, and tools based on viewpoint considerations. A survey of trends in multiparadigm modeling can be found in [1]. Trends and visions in multi-view modeling are also the topic of [19]. The latter paper also discusses pragmatics of MVM in the context of the Ptolemy tool. However formal aspects of MVM and algorithmic problems such as checking consistency are not discussed.

Implicitly, MVM is supported by multi-modeling languages such as UML, SysML, and AADL. For instance, AADL defines separate "behavior and error annexes" and having separate models in these annexes can result in inconsistencies. But capabilities such as conformance or consistency checking are typically not provided by the tools implementing these standards. Architectural consistency notions in a UML-like framework are studied in [6]. 
This work is a first step toward a formal and algorithm-supported framework for multiview modeling. A natural direction for future work is to study algorithmic problems such as consistency checking in a heterogeneous setting. Although the framework of Section 3 is general enough to capture heterogeneity, in this paper we restricted our attention to algorithmic MVM problems for discrete systems, as we feel that we first need a solid understanding of MVM in this simpler case.

Other directions for future work including investigating other types of abstraction functions, generalizing the methods developed in Section 5, e.g., so that $\subseteq,=, \supseteq$ can be arbitrarily combined, and studying algorithmic problems related to orthogonality.

\section{References}

1. V. Amaral, C. Hardebolle, G. Karsai, L. Lengyel, and T. Levendovszky. Recent advances in multi-paradigm modeling. In MODELS, pages 220-224. Springer, 2010.

2. A. Benveniste, B. Caillaud, A. Ferrari, L. Mangeruca, R. Passerone, and C. Sofronis. Multiple viewpoint contract-based specification and design. In FMCO, pages 200-225. Springer, 2008.

3. A. Bhave, B.H. Krogh, D. Garlan, and B. Schmerl. View consistency in architectures for cyber-physical systems. In ICCPS 2011, pages $151-160,2011$.

4. D. Broman, E.A. Lee, S. Tripakis, and M. Törngren. Viewpoints, Formalisms, Languages, and Tools for Cyber-Physical Systems. In MPM, 2012.

5. P. Cousot and R. Cousot. Systematic design of program analysis frameworks. In $P O P L$, pages 269-282. ACM, 1979.

6. R. M. Dijkman. Consistency in Multi-Viewpoint Architectural Design. PhD thesis, University of Twente, 2006.

7. L. Doyen, T. Henzinger, B. Jobstmann, and T. Petrov. Interface theories with component reuse. In EMSOFT, pages 79-88, 2008.

8. A. Finkelstein, D. Gabbay, A. Hunter, J. Kramer, and B. Nuseibeh. Inconsistency handling in multiperspective specifications. IEEE TSE, 20(8):569-578, 1994.

9. M. R. Garey and David S. Johnson. Computers and Intractability: A Guide to the Theory of NP-Completeness. W. H. Freeman, 1979.

10. P. Granger. Improving the results of static analyses programs by local decreasing iteration. In FSTTCS, pages 68-79, London, UK, UK, 1992. Springer-Verlag.

11. T. A. Henzinger and D. Nickovic. Independent implementability of viewpoints. In Monterey Workshop, volume 7539 of LNCS, pages 380-395. Springer, 2012.

12. ISO/IEC/IEEE 42010:2011. Systems and software engineering - Architecture description, the latest edition of the original IEEE Std 1471:2000, Recommended Practice for Architectural Description of Software-intensive Systems. IEEE and ISO, 2011.

13. J.-Y. Kao, N. Rampersad, and J. Shallit. On NFAs where all states are final, initial, or both. Theoretical Computer Science, 410(4749):5010 - 5021, 2009.

14. M. Persson, M. Törngren, et al. A Characterization of Integrated Multi-View Modeling for Embedded Systems. In EMSOFT, 2013.

15. A. Rajhans and B. H. Krogh. Heterogeneous verification of cyber-physical systems using behavior relations. In HSCC '12, pages 35-44. ACM, 2012.

16. A. Rajhans and B. H. Krogh. Compositional heterogeneous abstraction. In HSCC'13, pages 253-262. ACM, 2013.

17. Jan Reineke and Stavros Tripakis. Basic problems in multi-view modeling. Technical Report UCB/EECS-2014-3, EECS Department, University of California, Berkeley, Jan 2014.

18. S. Tripakis, B. Lickly, T. A. Henzinger, and E. A. Lee. A theory of synchronous relational interfaces. ACM Trans. on Progr. Lang. and Sys. (TOPLAS), 33(4), July 2011.

19. R. von Hanxleden, E. A. Lee, C. Motika, and H. Fuhrmann. Multi-view modeling and pragmatics in 2020. In 17th Intl. Monterey Workshop, LNCS, 2012. 